\title{
BOUNDARY LAYER FLOW OF REINER-PHILIPPOFF FLUIDS
}

\author{
T. Y. Na \\ Department of Mechanical Engineering, University of Michigan, Dearborn, MI 48128, U.S.A.
}

(Received 2 November 1993; accepted in final form 20 April 1994)

\begin{abstract}
An analysis is made of the boundary layer flow of Reiner-Philippoff fluids. This work is an extension of a previous analysis by Hansen and $\mathrm{Na}$ [A. G. Hansen and T. Y. Na, Similarity solutions of laminar, incompressible boundary layer equations of non-Newtonian fluids. ASME 67-WA/FE-2, presented at the ASME Winter Annual Meeting, November (1967)], where the existence of similar solutions of the boundary layer equations of a class of general non-Newtonian fluids were investigated. It was found that similarity solutions exist only for the case of flow over a $90^{\circ}$ wedge and, being similar, the solution of the non-linear boundary layer equations can be reduced to the solution of non-linear ordinary differential equations. In this paper, the more general case of the boundary layer flow of Reiner-Philippoff fluids over other body shapes will be considered. A general formulation is given which makes it possible to solve the boundary layer equations for any body shape by a finite-difference technique. As an example, the classical solution of the boundary layer flow over a flat plate, known as the Blasius solution, will be considered. Numerical results are generated for a series of values of the parameters in the Reiner-Philippoff model.
\end{abstract}

\section{NOMENCLATURE}

$C_{f} \quad$ skin friction coefficient

$f$ transformed dependent variable

$F \quad$ function relation $\tau$ and velocity gradient

$g$ transformed dependent variable

$L \quad$ reference length

$P(x) \quad$ function of body shapes

$\mathrm{Re} \quad$ Reynolds number

$u \quad$ velocity component in the $x$-direction

$U_{\infty} \quad$ velocity of the mainstream

$v \quad$ velocity component in the $y$-direction

$U_{e} \quad$ velocity at the edge of boundary layer

$x, y$ rectangular coordinates

\section{Greek symbols}

$\begin{array}{ll}\rho & \text { density } \\ \nu & \text { kinematic viscosity } \\ \mu & \text { dynamic viscosity } \\ \beta & \text { dimensionless parameter } \\ \gamma & \text { dimensionless parameter } \\ \psi & \text { stream function } \\ \tau & \text { shearing stress } \\ \zeta & \text { transformed independent variable } \\ \eta & \text { transformed independent variable }\end{array}$

$\begin{array}{ll}\text { Subscripts } & \\ \infty & \text { condition at infinity in the } y \text {-direction } \\ \boldsymbol{x} & \text { local } \\ w & \text { wall } \\ 0 & \text { reference condition. }\end{array}$

\section{INTRODUCTION}

The subject of boundary layer flows of non-Newtonian fluids has been a topic of investigation for some time. Some typical works on the topic are given in references [1]-[14]. Even though considerable progress has been made in our understanding of the flow phenomena, 
more works are needed to understand the effects of the various parameters involved in the non-Newtonian models and the formulation of an accurate method of analysis for any body shapes of engineering significance.

The problem is difficult because, in addition to the inherent difficulties in the solution of the boundary layer equations which are non-linear partial differential equations, the non-linear coupling of the shearing stress with the rate of strain of the various models, that is

$$
F\left(\tau, \frac{\partial u}{\partial y}\right)=0
$$

poses additional problems.

In the analysis of boundary layer problems, the class of solutions known as similarity solutions traditionally plays an important role because it is the only class of exact solutions for the boundary layer equations. For Newtonian fluids, it is well known that similarity solutions exist for the class of bodies known as the Falkner-Skan problems, which includes many practical geometries. The non-linear relation between the rate of shearing stress and the rate of strain in equation (1), however, places further restriction on the class of problems which can be solved by similarity transformations. The problem was investigated in the work of Hansen and $\mathrm{Na}$ [8], which found that for the boundary layer flows of nonNewtonian fluids, similarity solution exists only for the flow over a $90^{\circ}$ wedge, as shown in Fig. 1(a).

Even though very useful information can be revealed as to the various physical parameters on the boundary layer characteristics from the similarity solution, it is of limited engineering value since for practical purposes bodies other than a $90^{\circ}$ wedge will most likely be encountered. This points to the need for a general formulation and solution technique which can solve any problem of boundary layer flows of non-Newtonian fluids such as the Reiner-Philippoff fluid treated in this paper-a topic which seems to have been neglected in the literature. In this paper, we will therefore look beyond the similarity solution of the problem by considering shapes other than a $90^{\circ}$ wedge. A formulation is given in which the boundary layer equations are transformed to a form which are suitable for solution by a finite difference technique. The formulation is made into such a general form that boundary layer flows of any shape can be treated by entering the expression of the main stream velocity into a general function, $P(x)$. Numerical results will be presented in this paper for two examples, namely, the similarity solution of the flow over a $90^{\circ}$ wedge [Fig. 1(a)], and the non-similar solution of the flow over a semi-infinite flat plate with mainstream parallel to the plate [Fig. 1(b)]. The second example is known as the Blasius solution, which for the case of flow of Newtonian fluids is similar. Deviations from similarity solutions as shown in the present paper where non-Newtonian fluids are treated therefore shows clearly the effects of the various parameters involved in the model.

There are two reasons for studying this particular non-Newtonian fluid model. First, this model correctly represents a class of non-Newtonian fluids and yet there seems to be a lack of reported literature on the boundary layer flow of such fluids. Second, the present analysis introduces a method of formulation and solution which can be applied to the boundary

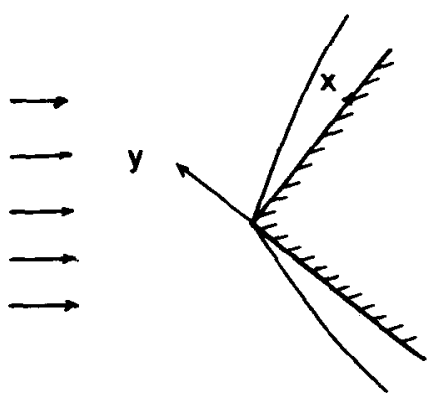

(a)

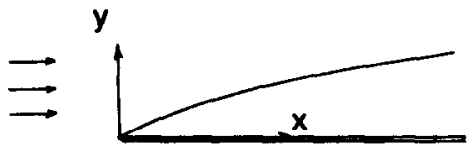

(b)

Fig. 1. Schematic diagrams of the examples. 
layer flow of any non-Newtonian fluid over any body shape in which the velocity gradient is expressed explicitly as a function of the shearing stress.

\section{ANALYSIS}

The governing differential equations for the boundary layer flow of a Reiner-Philippoff non-Newtonian fluid can be written as:

$$
\begin{gathered}
\frac{\partial u}{\partial x}+\frac{\partial v}{\partial y}=0 \\
\rho\left\{u \frac{\partial u}{\partial x}+v \frac{\partial u}{\partial y}\right\}=\frac{\partial \tau}{\partial y}+\rho U_{e} \frac{\mathrm{d} U_{e}}{\mathrm{~d} x}
\end{gathered}
$$

where the shearing stress, $\tau$, is related explicitly to the velocity gradient by

The boundary conditions are:

$$
\frac{\partial u}{\partial y}=\frac{\tau}{\mu_{\infty}+\frac{\mu_{0}-\mu_{\infty}}{1+\left\{\frac{\tau}{\tau_{s}}\right\}^{2}}}
$$

$$
\begin{aligned}
& y=0: u(x, 0)=0 ; v(x, 0)=0 \\
& y=\infty: u(x, \infty)=U_{e}(x) .
\end{aligned}
$$

Let us introduce the following dimensionless quantities

$$
\begin{aligned}
& \bar{x}=\frac{x}{L} ; \quad \bar{y}=\frac{y}{L} \sqrt{\operatorname{Re}} ; \quad \bar{u}=\frac{u}{U_{\infty}} ; \quad \bar{v}=\frac{v}{U_{\infty}} \sqrt{\operatorname{Re}} ; \\
& \bar{\tau}=\frac{\tau}{\rho U_{\infty}^{2}} \sqrt{\operatorname{Re}} ; \quad \bar{\mu}=\frac{\mu_{0}}{\mu_{\infty}} ; \quad \operatorname{Re}=\frac{U_{\infty} L}{v}
\end{aligned}
$$

and a stream function, $\bar{\psi}$, such that

$$
\bar{u}=\frac{\partial \bar{\psi}}{\partial \bar{y}} ; \quad \bar{v}=-\frac{\partial \bar{\psi}}{\partial \bar{x}} .
$$

Equations (2)-(6) become

$$
\begin{gathered}
\frac{\partial \bar{\psi}}{\partial \bar{y}} \frac{\partial^{2} \bar{\psi}}{\partial \bar{x} \partial \bar{y}}-\frac{\partial \bar{\psi}}{\partial \bar{x}} \frac{\partial^{2} \bar{\psi}}{\partial \bar{y}^{2}}=\frac{\partial \bar{\tau}}{\partial \bar{y}}+\bar{U}_{e} \frac{\mathrm{d} \bar{U}_{e}}{\mathrm{~d} \bar{x}} \\
\frac{\partial \bar{u}}{\partial \bar{y}}=\frac{\bar{\tau}}{1+\frac{\bar{\mu}_{0}-1}{1+\left\{\frac{\bar{\tau} \bar{\tau}_{s}}{\bar{\tau}_{s}}\right\}}}
\end{gathered}
$$

subject to the boundary conditions

$$
\begin{aligned}
& \bar{y}=0: \frac{\partial \bar{\psi}(\bar{x}, 0)}{\partial \bar{y}}=0 ; \quad \frac{\partial \bar{\psi}(\bar{x}, 0)}{\partial \bar{x}}=0 \\
& \bar{y}=\infty: \frac{\partial \bar{\psi}(\bar{x}, \infty)}{\partial \bar{y}}=\bar{U}_{e} .
\end{aligned}
$$

Equations (9)-(12) represent a system of non-linear partial differential equations, the solution of which is quite difficult. One major simplification can be achieved by using the similarity transformation where the system of non-linear partial differential equations are reduced to a system of ordinary differential equations. Such transformations are limited to some special forms of the mainstream velocities. For the case of boundary layer flows of general non-Newtonian fluid, it was proved by Hansen and $\mathrm{Na}[8]$ that similarity solutions 
exist only if the mainstream velocity is given by

$$
\bar{U}_{e}(\bar{x})=\bar{x}^{1 / 3}
$$

which corresponds to the boundary layer flow over a $90^{\circ}$ wedge, as shown in Fig. 1(a). For other shapes, the flow is non-similar and a transformation will be introduced in this paper to reduce equations (9)-(12) to a form which can be solved by a finite-difference method.

\section{Similarity solution-flow over a $90^{\circ}$ wedge}

For this case, the mainstream velocity is given by equation (13) and a similarity transformation can be introduced as:

$$
\eta=\frac{\bar{y}}{\bar{x}^{1 / 3}} ; \quad f(\eta)=\frac{\bar{\psi}}{\bar{x}^{2 / 3}} ; \quad g(\eta)=\bar{\tau} .
$$

Equations (9)-(12) become

$$
\begin{gathered}
g^{\prime}+\frac{2}{3} f f^{\prime \prime}-\frac{1}{3}\left(f^{\prime}\right)^{2}+\frac{1}{3}=0 \\
g=f^{\prime \prime} \frac{g^{2}+\bar{\mu}_{0} \gamma}{\gamma+g^{2}}
\end{gathered}
$$

subject to the boundary conditions

$$
f(0)=0 ; \quad f^{\prime}(0)=0 ; \quad f^{\prime}(\infty)=1 .
$$

The skin friction coefficient is defined as

$$
C_{f x}=\frac{\tau_{w}}{\frac{1}{2} \rho U_{e}^{2}}=\frac{2 \bar{\tau}_{w}}{\bar{U}_{e}^{2}} \sqrt{\frac{v}{U_{e} L}}
$$

from which,

$$
\frac{1}{2} C_{f x} \sqrt{R e_{x}}=\bar{\tau}_{w} \sqrt{\frac{\bar{x}}{U_{e}^{3}}} .
$$

For boundary layer flows over a $90^{\circ}$ wedge, the mainstream velocity is given by equation (13). Equation (18) becomes

$$
\frac{1}{2} C_{f x} \sqrt{\operatorname{Re} x}=\bar{\tau}_{w}=g(\zeta, 0) .
$$

\section{Non-similar solution}

For the general case in which the boundary layer over any body shape is to be analyzed, a general transformation is introduced as follows:

$$
\zeta=\bar{x} ; \quad \eta=\sqrt{\bar{U}_{e}} \frac{\bar{y}}{\sqrt{\bar{x}}} ; \quad f(\zeta, \eta)=\frac{\bar{\psi}}{\sqrt{\bar{x} \bar{U}_{e}}} ; \quad g(\zeta, \eta)=\bar{\tau} \sqrt{\frac{\bar{x}}{\bar{U}_{e}^{3}}} .
$$

Under this transformation, equations (9)-(12) become

$$
\begin{gathered}
g^{\prime}+\frac{P(\zeta)+1}{2} f f^{\prime \prime}+P(\zeta)\left\{1-\left[f^{\prime}\right]^{2}\right\}=\zeta\left\{f^{\prime} \frac{\partial f^{\prime}}{\partial \zeta}-f^{\prime \prime} \frac{\partial f}{\partial \zeta}\right\} \\
g=f^{\prime \prime} \frac{\bar{\mu}_{0} \gamma \zeta+\bar{U}_{e}^{3} g^{2}}{\gamma \zeta+\bar{U}_{e}^{3} g^{2}}
\end{gathered}
$$

subject to the boundary conditions

where

$$
f(0)=0 ; \quad f^{\prime}(0)=0 ; \quad f^{\prime}(\infty)=1,
$$

$$
P(\zeta)=\frac{\zeta}{\bar{U}_{e}} \frac{\mathrm{d} \bar{U}_{e}}{\mathrm{~d} \zeta}
$$


The skin friction coefficient can be defined as

$$
C_{f x}=\frac{\tau_{w}}{\frac{1}{2} \rho U_{e}^{2}}
$$

We therefore obtain

$$
\frac{1}{2} C_{f x} \sqrt{R e_{x}}=\frac{1}{2} \frac{\tau_{w}}{\frac{1}{2} \rho U_{e}^{2}} \sqrt{\frac{U_{e} x}{v}}=\bar{\tau}_{w} \sqrt{\frac{\bar{x}}{\bar{U}_{e}^{3}}}=g(\zeta, 0) .
$$

Equations (21)-(23) are very general since for a given body shape, the mainstream velocity can be substituted into the function $P(\zeta)$ and equation (22) and the solution of the non-linear partial differential equations, equations (21) and (22), subject to the boundary conditions [equation (23)], can be solved in principle by a finite-difference method commonly used in the solution of boundary layer equations of Newtonian fluids. Details of the method will not be given here. The interested reader can refer to, for example, reference [15]. The additional difficulty results from the relation between $g$ and $f^{\prime \prime}$ in equation (22). At any $\zeta$-station, an iterative process in which the value of $g$ is first approximated by its value at the previous $\zeta$-station [equation (21)], subject to boundary conditions (23), is then solved by the finite-difference method. A new value of $g$ is then calculated by writing equation (22) as

$$
g_{n+1}=f_{n}^{\prime \prime} \frac{\bar{\mu}_{0} \gamma \zeta+\bar{U}_{e}^{3} g_{n}^{2}}{\gamma \zeta+\bar{U}_{e}^{3} g_{n}^{2}}
$$

where $n$ represents the number of iteration. The solution of equation (21) is then repeated until convergence in $g$ is reached.

\section{RESULTS AND DISCUSSIONS}

Numerical solutions are generated for two examples. The first example considers the similar case of the flow over a $90^{\circ}$ wedge. Numerical solutions of the skin friction expression, equation (19), for a range of $\mu_{0} / \mu_{\infty}$ and for $\gamma=0.1,0.5$ and 2, respectively, are shown in Fig. 2. As compared with the case of the Newtonian fluids which is constant at $f^{\prime \prime}(0)=0.75745$, the friction is seen to be larger for Reiner-Philippoff fluids. The difference increases for larger $\gamma \mathrm{s}$ and/or $\mu_{0} / \mu_{\infty}$. For Reiner-Philippoff fluids with smaller values of $\gamma$ and/or $\mu_{0} / \mu_{\infty}$, the friction coefficient becomes closer to the value of a Newtonian fluid.

As a second example, a non-similar case will be considered. The problem of the uniform flow over a semi-infinite flat plate, known as Blasius flow, will be selected. The boundary layer flow over a semi-infinite flat plate where the mainstream velocity is uniform and parallel to the plate is known as the Blasius problem which, for Newtonian flows, is similar

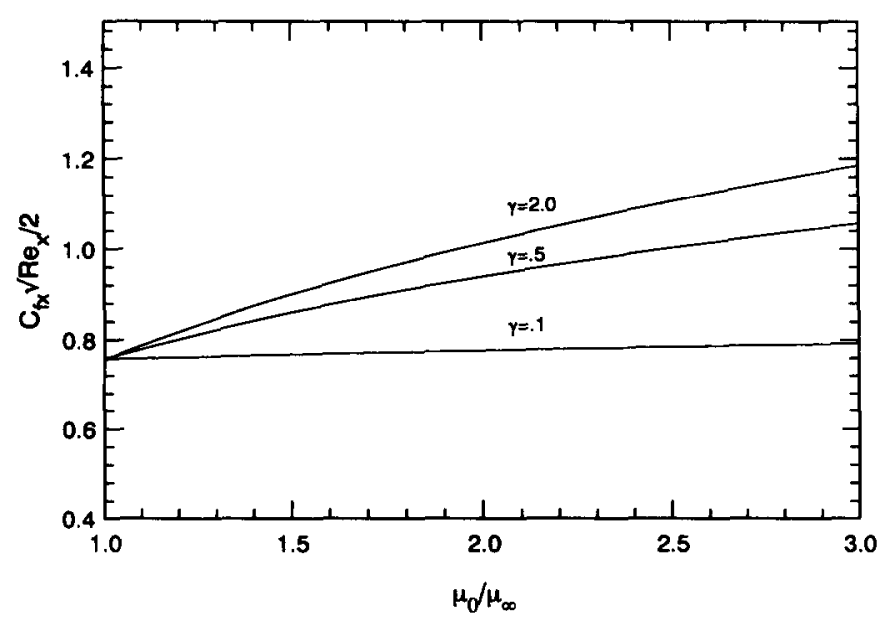

Fig. 2. $C_{f x} \sqrt{ } \operatorname{Re} e_{x} / 2$ vs $\mu_{0} / \mu_{\infty}$. 
and the boundary layer equations can be transformed to an ordinary differential equation. The non-Newtonian nature of the present problem makes the Blasius problem non-similar. Deviation of the present problem from the similarity solution of the classic Blasius solution therefore gives the effects of the two parameters in the Reiner-Philippoff model on the flow characteristics. The results are shown in Figs 3 and 4.

For flow over a semi-infinite flat plate with mainstream flow parallel to the plate,

$$
\bar{U}_{e}(\zeta)=1 \text {. }
$$

The skin friction coefficient expression, equation (25), becomes

$$
{ }_{2}^{1} C_{f x} \sqrt{R e_{x}}=\sqrt{\bar{x}} \bar{\tau}_{w}=g(\zeta, 0) .
$$

For Newtonian fluids, the skin friction coefficient, equation (28), equals 0.33206 for all $\zeta \mathrm{s}$ since the flow is similar. It is represented by a horizontal line in both Figs 3 and 4 . The trend of the effects of the two parameters, namely, $\gamma$ and $\mu_{0} / \mu_{\infty}$, are the same as in Fig. 2. The order-of-magnitude of the deviation from Newtonian behavior shows clearly that for Reiner-Philippoff fluids, solutions based on Newtonian solution are no longer applicable and the present solution procedure offers an efficient way of solving such problems.

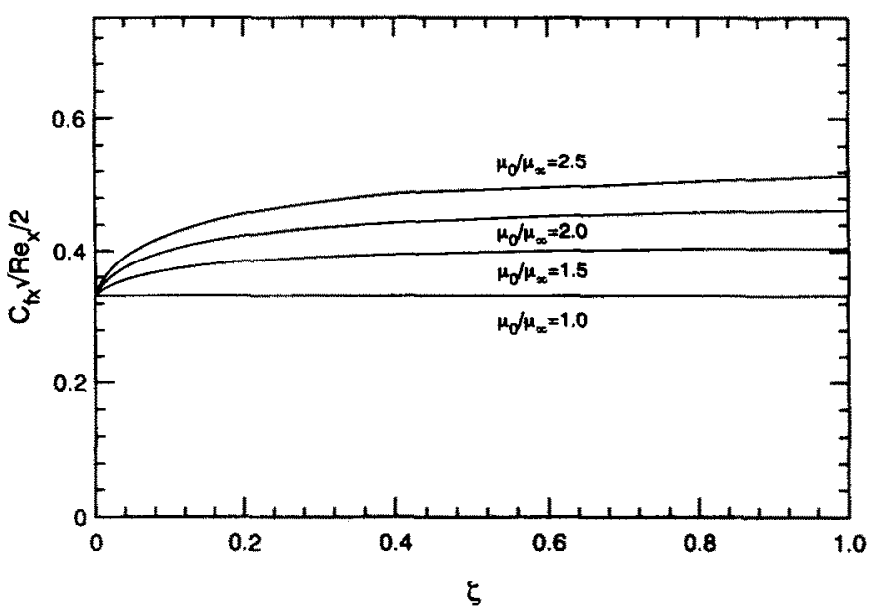

Fig. 3. $C_{f x} \sqrt{ } \operatorname{Re} x / 2$ vs $\zeta(\gamma=0.5)$.

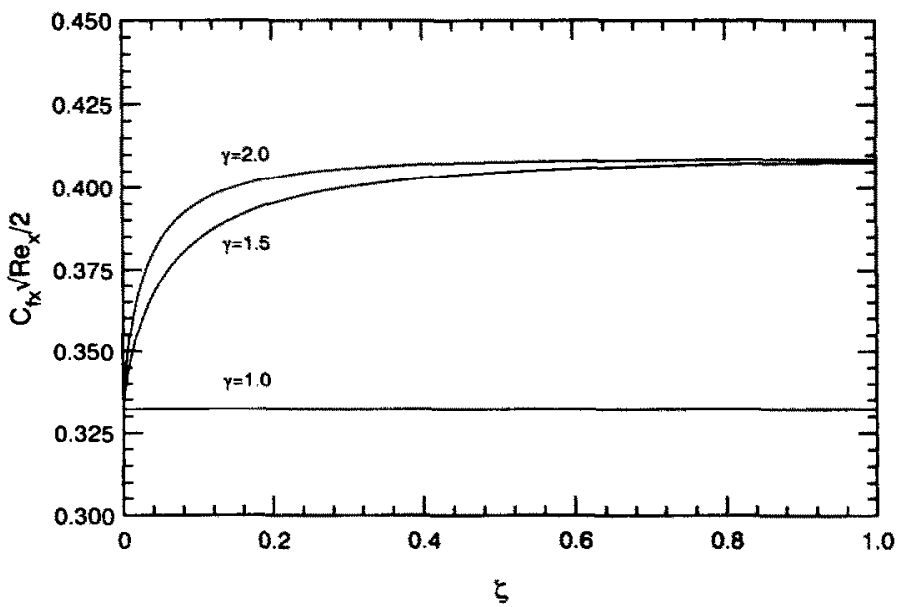

Fig. 4. $C_{f \times \sqrt{ }} / \operatorname{Re}_{x} / 2$ vs $\zeta\left(\mu_{0} / \mu_{\infty}=1.5\right)$. 


\section{CONCLUSIONS}

An analysis is made of the boundary layer flow of Reiner-Philippoff fluids over bodies other than a $90^{\circ}$ wedge. A general formulation is given in which the boundary layer equations for any body shape can be solved by a finite-difference technique. For the similar case, solutions of the flow over a $90^{\circ}$ wedge are generated for a family of values of the parameters in the model. For the non-similar case, the classical solutions of the boundary layer flow over a flat plate-known as the Blasius solution-are generated which, due to the non-Newtonian nature of the fluids, are now non-similar. Numerical results are generated for a series of values of the parameters in the model.

For any other body shapes, the same formulation can be used and the only change is in the two functions, namely, $P(\zeta)$ and equation (22) by substituting into these functions the mainstream velocity for that particular geometry. The present analysis therefore provides a very useful tool in the boundary layer flow not only of Reiner-Philippoff fluids but other fluids which can be defined by equation (1).

\section{REFERENCES}

1. A. Acrivos, M. J. Shah and E. E. Peterson, Momentum and heat transfer in laminar boundary layer flows of non-Newtonian fluids past external surfaces. AIChE J. 6, 312 (1960).

2. A. Acrivos, M. J. Shah and E. E. Peterson, On the solution of the two-dimensional boundary layer flow equations for a non-Newtonian power-law fluid. Chem. Engng Sci. 20, 101 (1965).

3. G. D. Bizzell and J. C. Alattery, Non-Newtonian boundary layer flow. Chem. Engng Sci. 17, 777 (1962).

4. N. Hayasi, Similarity of two-dimensional and axisymmetric boundary layer flows of non-Newtonian fluids. J. Fluid Mech. 23, 293-303 (1965).

5. J. N. Kapur and R. C. Scrivstava, Similar solutions of the boundary layer equations for power-law fluids. ZAMP 14, 383 (1963).

6. S. Y. Lee and W. F. Ames, Similar solutions for non-Newtonian fluids. AIChE J. 12, 700 (1966).

7. W. R. Schowalter, The application of boundary layer theory to power law pseudoplastic fluids: similar solutions. AIChE J. 6, 24 (1960).

8. A. G. Hansen and $\mathrm{T}$. Y. Na, Similarity solutions of laminar, incompressible boundary layer equations of non-Newtonian fluids. ASME 67-WA/FE-2, presented at the ASME Winter Annual Meeting, November (1967).

9. D. W. Beard and K. Walters, Elastico-viscous boundary layer flows-I. Two-dimensional flows near a stagnation point. Proc. Camb. Phil. Soc. 60, 667-674 (1964).

10. M. M. Denn, Boundary layer flows of a class of elastic liquids. Chem. Engng Sci. 22, 395-405 (1967).

11. R. W. Seth, Solution of a viscoelastic boundary layer equations by orthogonal collocation. $J$. Engng Math. 8, 89-92 (1974).

12. K. R. Rajagopal, T. Y. Na and A. S. Gupta, Flow of a viscoelastic fluid over a stretching sheet. Rheol. Acta 23, 213-215 (1984).

13. A. S. Gupta, K. R. Rajagopal and T. Y. Na, Falkner-Skan flow of a second-order fluid. Int. J. Non-Linear Mech. 18, 313-320 (1983).

14. W. H. H. Banks and M. B. Zaturska, Eigensolutions in boundary layer flow adjacent to a stretching sheet. IMA J. appl. Mathematics 36, 263-273 (1986).

15. T. Y. Na, Computational Methods in Engineering Boundary Value Problems. Academic Press, New York (1979). 